\title{
SUMS OF NORMAL ENDOMORPHISMS
}

\author{
R. H. BRUCK ${ }^{1}$
}

1. Introduction. The main object of this note is to prove the following:

Theorem. Let $(L,+)$ be the additive loop generated by the set of all normal endomorphisms of a loop $G$. A necessary and sufficient condition that $(L,+, \cdot)$ be an (ordinary, associative) ring is that $G$ be powerassociative.

It should be noted that the definition (introduced in [1]) of a normal endomorphism of a loop $G$ is radically different from the usual definition for groups. Nevertheless (as shown in [2]) the two definitions are equivalent when $G$ is a group. In particular, the present theorem generalizes one of Heerema [3]. We may add that in [2], by assuming that the loop $G$ was Moufang, we were able to be much more explicit than at present about the properties of the ring $(L,+, \cdot)$. For example, if $G$ is merely power-associative and if $\theta$ is an element of $L$ which happens to be an endomorphism, we can answer neither of the following questions: (i) Is the complement $1-\theta$ an endomorphism? (ii) Is $\theta$ semi-normal in the sense of [2]?

The definition of a normal endomorphism involves the concept of a purely nonabelian (p.n.a.) loop word; this will be discussed in $\$ 2$. Roughly speaking, p.n.a. loop words are the analogues for loops of the higher commutator forms introduced by Philip Hall in the theory of groups, although an inductive definition of p.n.a. loop words as "higher commutator-associator forms" does not seem profitable at present. In particular, the following simple lemma, on which the proof of the theorem hinges, could be generalized to deal with the various terms of the lower central series or derived series of a loop:

Lemma 1. Let $H$ be a loop generated by a (nonempty) subset $A$. Then the following condition (C) is both necessary and sufficient in order that $H$ be an abelian group:

(C) $W_{n}\left(a_{1}, \cdots, a_{n}\right)=1$ for every positive integer $n$, every normalized p.n.a. loop word $W_{n}$, and all choices of $a_{1}, \cdots, a_{n}$ in $A$.

Indeed, our proof of Lemma 1 could easily be rearranged to prove a

Presented to the Society, January 22, 1959; received by the editors November 14, 1958.

1 This research was supported by a grant from the National Science Foundation. 
stronger statement: The elements $W_{n}\left(a_{1}, \cdots, a_{n}\right)$ generate the commutator-associator subloop, $H^{\prime}$, of $H$.

2. Preliminaries. By a loop word $W_{n}$ we mean an element of the free loop $F$ on $n$ free generators $X_{1}, \cdots, X_{n}$. If $G$ is a loop and $a_{1}, \cdots, a_{n}$ are elements of $G, W_{n}\left(a_{1}, \cdots, a_{n}\right)$ denotes the image of $W_{n}$ under the (uniquely defined) homomorphism of $F$ into $G$ which maps $X_{i}$ into $a_{i}$ for $i=1,2, \cdots, n$.

As shown in [2], each of the following properties of a loop word $W_{n}$ implies the others:

(i) $W_{n}$ is an element of the commutator-associator subloop $F^{\prime}$.

(ii) $W_{n}$ vanishes on every abelian group.

(iii) If $G$ is a loop with centre $Z$ then

$$
W_{n}\left(x_{1} c_{1}, \cdots, x_{n} c_{n}\right)=W_{n}\left(x_{1}, \cdots, x_{n}\right)
$$

for all $x_{i}$ in $G$ and $c_{i}$ in $Z$.

A loop word $W_{n}$ is called purely nonabelian (p.n.a.) if it satisfies the equivalent properties (i)-(iii).

Now we need some new definitions. Fix attention on a generator $X_{i}$ of the free loop $F$ and let $\theta_{i}$ be the (idempotent) endomorphism of $F$ which maps $X_{i}$ into the identity element, 1 , of $F$ and fixes $X_{j}$ for each $j \neq i$. Then, if $W_{n}$ is in $F$, we call $X_{i}$ a nonessential argument of $W_{n}$ if $W_{n} \theta_{i}=W_{n}$, and an essential argument of $W_{n}$ if $W_{n} \theta_{i} \neq W_{n}$. Note that a loop word is an element of the free subloop on its essential arguments.

A loop word $W_{n}$ is called normalized provided that $W_{n} \theta_{i}=1$ for $i=1,2, \cdots, n$. We shall need the following refinement: $W_{n}$ is called essentially normalized if $W_{n} \theta_{i}=1$ whenever $X_{i}$ is an essential arument of $W_{n}$. In other words, $W_{n}$ is essentially normalized if it is normalized when considered as an element of the free loop on its essential arguments.

Lemma 2. If $W_{n}$ is a p.n.a. loop word, there exists a finite set $\mathfrak{\Im}_{n}$ of essentially normalized p.n.a. loop words such that $W_{n}$ is in the set $\mathfrak{S}_{n}^{*}$ defined inductively as follows:

(i) $\mathfrak{S}_{n} \subset \mathfrak{S}_{n}^{*}$.

(ii) If $A_{n}, B_{n}$ are in $\mathfrak{S}_{n}^{*}$, then $A_{n} B_{n}$ is in $\mathfrak{S}_{n}^{*}$.

Proof. First we note that, if $n=1$, every loop word $W_{1}$ is normalized and hence essentially normalized. Therefore, if $n=1$, we may define $\widetilde{S}_{1}$ to consist of the given p.n.a. word $W_{1}$.

Next we consider the case $n>1$ and assume inductively that the lemma is true for $n-1$. We define loop words $A_{n}, B_{n}$ by

$$
A_{n}=W_{n} \theta_{n}, \quad W_{n}=A_{n} B_{n} .
$$


We observe first that $A_{n}, B_{n}$ are p.n.a. loop words. In addition, $X_{n}$ is a nonessential argument of $A_{n}$; consequently $A_{n}$ may be considered as an element $A_{n-1}$ of the free loop on $X_{1}, \cdots, X_{n-1}$. Therefore, by our inductive assumption, $A_{n}$ may be built up in the manner of the lemma from a finite set of essentially normalized p.n.a. words each of which has $X_{n}$ as a nonessential argument. On the other hand, $B_{n} \theta_{n}=1$, so $B_{n}$ is "normalized in $X_{n}$." Next we define loop words $C_{n}, D_{n}$ by

$$
C_{n}=B_{n} \theta_{n-1}, \quad B_{n}=C_{n} D_{n} .
$$

Again, $C_{n}, D_{n}$ are p.n.a. Moreover, the inductive assumption applies to $C_{n}$, while $D_{n}$ is normalized both in $X_{n}$ and in $X_{n-1}$. If $n>2$, we proceed in the same manner to "split" $D_{n}$ by means of $\theta_{n-2}$. Since the indicated process must come to an end in a finite number of steps, the truth of Lemma 2 is now clear.

Next we must consider the set $M$ of all single-valued mappings of a multiplicative loop $G$ into itself. The product, $\alpha \beta$, and the sum, $\alpha+\beta$, of two elements $\alpha, \beta$ of $M$ are defined by

$$
x(\alpha \beta)=(x \alpha) \beta, \quad x(\alpha+\beta)=(x \alpha)(x \beta)
$$

for all $x$ in $G$. We note that $(M,+)$ is a loop. In addition, if $(L,+)$ is a subloop of $(M,+)$ and $x$ is any fixed element of $G$, the mapping $\alpha \rightarrow x \alpha$ induces a homomorphism of $(L,+)$ upon a subloop $x L$ of $G$. As one consequence, if $W_{n}$ is a loop word,

$$
x W_{n}\left(\alpha_{1}, \cdots, \alpha_{n}\right)=W_{n}\left(x \alpha_{1}, \cdots, x \alpha_{n}\right)
$$

for all $x$ in $G$ and $\alpha_{1}, \cdots, \alpha_{n}$ in $(M,+)$. Moreover

$$
\alpha(\beta+\gamma)=\alpha \beta+\alpha \gamma
$$

for all $\alpha, \beta, \gamma$ in $M$, and

$$
(\beta+\gamma) \theta=\beta \theta+\gamma \theta
$$

for all $\beta, \gamma$ in $M$ precisely when $\theta$ is an endomorphism of $G$.

And, finally, we must recall the definition of a normal endomorphism. An endomorphism $\theta$ of a loop $G$ is normal provided that

$$
W_{n}\left(x_{1}, \cdots, x_{n}\right) \theta=W_{n}\left(x_{1} \theta, x_{2}, \cdots, x_{n}\right)
$$

for every choice of a positive integer $n$, a normalized p.n.a. word $W_{n}$, and elements $x_{1}, \cdots, x_{n}$ of $G$. When $\theta$ is normal, (2.5) also holds with the right hand side replaced by any one of

$$
W_{n}\left(x_{1}, x_{2} \theta, \cdots, x_{n}\right), \cdots, W_{n}\left(x_{1}, x_{2}, \cdots, x_{n} \theta\right) .
$$


3. Proof of Lemma 1 . Let $H$ be a loop generated by a subset $A$. If $H$ is an abelian group, condition (C) obviously holds for every p.n.a. loop word $W_{n}$. On the other hand, if (C) holds for every normalized p.n.a. loop word $W_{n}$, then, by Lemma 2 and the definition of "essentially normalized," (C) also holds for every p.n.a. loop word $W_{n}$ (not necessarily normalized).

We shall use this strengthened form of (C) to show that $H^{\prime}=1$, where $G^{\prime}$ denotes the commutator-associator subloop of a loop $G$. If $h$ is an element of $H^{\prime}$, there exists a finite set $a_{1}, \cdots, a_{n}$ of elements of $A$ such that $h$ is in $K^{\prime}$ where $K$ is the subloop generated by $a_{1}, \cdots, a_{n}$. Let $F$ be the free loop on $X_{1}, \cdots, X_{n}$, and let $\theta$ be the homomorphism such that $X_{i} \theta=a_{i}$ for $i=1,2, \cdots, n$. Then $\theta$ maps $F$ upon $K$ and $F^{\prime}$ upon $K^{\prime}$. Moreover, by definition, an element $W_{n}$ of $F$ is in $F^{\prime}$ precisely when $W_{n}$ is p.n.a. Hence $K^{\prime}=1$, so that $h=1$ and therefore $H^{\prime}=1$. That is, $H$ is an abelian group. This completes the proof of Lemma 1.

4. Proof of the theorem. Let $G$ be a loop, let $A$ be the set of all normal endomorphisms of $G$ and let $(L,+)$ be the additive loop generated by $A$. We note that $A A \subset A$ and that the identity mapping, $I$, of $G$ is in $A$. The zero mapping, 0 , defined by

$$
x 0=1 \text {, }
$$

all $x$ in $G$,

is also in $A$ and is in fact the additive identity element of $L$. Now let $x$ be any element of $G, W_{n}$ be any normalized p.n.a. loop word, and $\phi_{1}, \cdots, \phi_{n}$ be any $n$ elements of $A$. Then

$$
x W_{n}\left(\phi_{1}, \cdots, \phi_{n}\right)=W_{n}\left(x \phi_{1}, \cdots, x \phi_{n}\right),
$$

by $(2.2)$, and

$$
W_{n}\left(x \phi_{1}, \cdots, x \phi_{n}\right)=W_{n}(x, \cdots, x) \phi_{1} \cdots \phi_{n}
$$

by the normality of $\phi_{1}, \cdots, \phi_{n}$.

If $G$ is power-associative then, by Lemma 1 applied to the subloop generated by $x$, the right hand side of (4.3) reduces to $1 \phi_{1} \cdots \phi_{n}=1$. Accordingly, (4.2) yields

$$
W_{n}\left(\phi_{1}, \cdots, \phi_{n}\right)=0
$$

for all $\phi_{1}, \cdots, \phi_{n}$ in $A$. But then, by Lemma 1 applied to the additive loop $(L,+),(L,+)$ is an abelian group. Conversely, if $(L,+)$ is an abelian group, (4.4) holds in particular when each $\phi_{i}$ is $I$. In this case, (4.2) yields

$$
W_{n}(x, \cdots, x)=1
$$


for every $x$. Therefore each element of $G$ generates an abelian group. We have now shown that $(L,+)$ is an abelian group precisely when $G$ is power-associative. In particular, then, $G$ must be power-associative if $(L,+, \cdot)$ is a ring.

We assume henceforth that $(L,+)$ is an abelian group.

Since $A A \subset A$, two simple arguments based on (2.4), (2.3) allow us to prove, respectively, that $L A \subset L$ and that $L L \subset L$. (For details see [2].) Hence $(L, \cdot)$ is a semigroup. In addition, for each fixed pair $\alpha, \beta$ of elements of $L$, the mapping

$$
\gamma \rightarrow(\alpha+\beta) \gamma-\alpha \gamma-\beta \gamma
$$

is an endomorphism of $(L,+)$ which sends $A$ into 0 . Hence

$$
(\alpha+\beta) \gamma=\alpha \gamma+\beta \gamma
$$

for all $\alpha, \beta, \gamma$ in $L$. In view of (2.3), (4.5), $(L,+, \cdot)$ is clearly a ring. This completes the proof of the theorem.

\section{REFERENCES}

1. R. H. Bruck, A survey of binary systems, Ergebnisse der Mathematik und Ihrer Grenzgebiete, Neue Folge, Heft 20, Berlin-Göttingen-Heidelberg, Springer-Verlag, 1958; Math. Rev. vol. 20 (1959) p. 13.

2. —- Normal endomorphisms, Illinois J. Math. to appear.

3. Nicholas Heerema, Sums of normal endomorphisms, Trans. Amer. Math. Soc. (1957) pp. 137-143; Math. Rev. vol. 18, p. 559.

UNIVERSITY OF WISCONSIN 